\title{
QPOs in Microquasars: the Spin Problem
}

\author{
Gabriel Török ${ }^{1,3}$, Marek A. Abramowicz ${ }^{1,2,3}$, \\ Zdeněk Stuchlík ${ }^{1}$ and Eva Šrámková ${ }^{1,3}$ \\ ${ }^{1}$ Institute of Physics, Faculty of Philosophy and Science, Silesian University in Opava, \\ Bezručovo nám. 13, CZ-74601 Opava, Czech Republic. \\ email: terek@volny.cz, zdenek.stuchlik@fpf.slu.cz, sram_eva@centrum.cz \\ ${ }^{2}$ Department of Physics, Göteborg University, S-412 96 Göteborg, Sweden. \\ email: marek@fy.chalmers.se \\ ${ }^{3}$ Copernicus Astronomical Centre, Polish Academy of Sciences, PL-00 716 Warszawa, \\ Bartycka 18, Poland
}

\begin{abstract}
.
In Galactic microquasars with double-peak $\mathrm{kHz}$ quasi-periodic oscillations (QPOs), the ratio of the two frequencies is $3: 2$. This supports the suggestion that double-peak $\mathrm{kHz}$ QPOs are due to a non-linear resonance between two modes of accretion disk oscillations. For the microquasars with known mass, we briefly compare the black hole spin estimates based on the orbital resonance model with the recently reported spin predictions obtained by fitting the spectral continua. Results of these two approaches are not in good agreement. We stress that if the spectral fit estimates are accurate and can be taken as referential (which is still questionable), the disagreement between the predicted and referential values would represent a rather generic problem for any relativistic QPO model, as no spin influence would appear in the observed $1 / M$ scaling of the QPO frequencies. The epicyclic frequencies relevant in these models are often considered to be equal to those of a test particle motion. However modifications of the frequencies due to disc pressure or other non-geodesic effects may play an important role, and the inaccuracy introduced in the spin estimates by the test particle approximation could be crucial.
\end{abstract}

Keywords. Black hole physics, X-rays: binaries

\section{Estimating the black hole spin from the resonance models}

The resonance model (Kluźniak \& Abramowicz 2000) explains the twin peak QPOs as being caused by a 3:2 non-linear resonance between two global modes of oscillations in accretion flow in strong gravity. The modes in resonance are often assumed to be the epicyclic modes. The orbital resonance model (see Kluźniak \& Abramowicz 2003) demonstrates that fluid accretion flows admit two linear quasi-incompressible modes of oscillations, radial and vertical, with corresponding eigenfrequencies equal to the radial and vertical epicyclic frequencies for free particles (Aliev \& Galtsov 1981; Nowak \& Lehr 1998). According to the resonance hypothesis, the two modes in resonance have eigenfrequencies $\nu_{\mathrm{r}}$ (radial epicyclic frequency) and $\nu_{\mathrm{v}}$ (vertical epicyclic frequency $\nu_{\theta}$ or Keplerian frequency $\nu_{\mathrm{K}}$ ). Several resonances of this kind are possible and have been discussed (see, e.g., Abramowicz \& Kluźniak 2004).

Formulae for the Keplerian $\nu_{\mathrm{K}}$ and the epicyclic frequencies $\nu_{\mathrm{r}}$ and $\nu_{\theta}$ in the field of a Kerr black hole with mass $M$ and spin $a$ are well known, and have the general form

$$
\nu=\left(\frac{G M_{0}}{r_{G}^{3}}\right)^{1 / 2} f_{\mathrm{i}}(x, a) \doteq 32.3\left(\frac{M_{0}}{M_{\odot}}\right) f_{\mathrm{i}}(x, a) \mathrm{kHz}, \quad \mathrm{i} \in \mathrm{K}, \mathrm{r}, \theta
$$

where $f_{\mathrm{i}}(x, a)$ are functions of a dimensionless black hole spin $a$ and a dimensionless radial 
Table 1. Spin estimates from the resonance models for microquasars (for details and other considered resonances see, e.g., Török et al. 2005; Török 2005). Spin intervals correspond to the $1 \sigma$ uncertainty in mass, the small error resulting from uncertainty of the frequency measurement is for XTE 1550-564 (GRO 1655-40, GRS 1915+105) up to 0.03 (0.01, 0.05).

\begin{tabular}{lrrrr}
\hline Model for & \multicolumn{5}{c}{ Interval of possible spin a relevant for } \\
& $1550-564$ & $1655-40$ & $1655-40^{*}$ & $1915+105$ \\
\hline $3: 2\left[\nu_{\theta}, \nu_{r}\right]$ & $+0.89-+0.99$ & $+0.96-+0.99$ & $+0.88-+0.93$ & $+0.69-+0.99$ \\
$2: 1\left[\nu_{\theta}, \nu_{r}\right]$ & $+0.12-+0.42$ & $+0.31-+0.42$ & $+0.10-+0.25$ & $-0.41-+0.44$ \\
$3: 1\left[\nu_{\theta}, \nu_{r}\right]$ & $+0.32-+0.59$ & $+0.50-+0.59$ & $+0.31-+0.44$ & $-0.15-+0.61$ \\
\hline
\end{tabular}

* The two columns for GRO 1655-40 indicate numbers following from the two different mass analysis - Beer \& Podsiadlowski (2002) vs. Greene et al. (2001). Note that while the spin estimates from the $3: 2$ parametric resonance is for both the cases similar $(a \approx 0.9)$, for the other models, the given mass range implies a large range of spins.

coordinate $x=r / M$. For a $n: m$ orbital resonance, the dimensionless resonance radius $x_{\mathrm{n}: \mathrm{m}}$ is determined as a function of spin $a$ by an equation $\mathrm{n} \nu_{\mathrm{r}}=\mathrm{m} \nu_{\mathrm{v}}\left(\nu_{\mathrm{v}}=\nu_{\theta}\right.$ or $\left.\nu_{\mathrm{K}}\right)$ $\dagger$. Thus, from the observed frequencies and from the estimated mass, one can determine the relevant spin (Abramowicz \& Kluźniak 2001; Török et al. 2005). We summarize the spin estimates for the three microquasars in Table 1.

\section{Comparison with the fits of spectral continua}

Except for one case, all the resonances considered in Török et al. (2005) are consistent with reasonable values of the black hole spin covering the range $a \in(0,1)$. In particular, the 3:2 epicyclic parametric (internal) resonance model, supposed to be the most natural one in Einstein gravity (Horák 2005), implies the spin $a \sim 0.9$.

The most recent results of the spectral fits correspond for GRO 1655-40 to the spin $a \in(0.65,0.75)$, and for GRS $1915+105$ to $a>0.98$ (McClintock et al. 2006). Obviously, the value for GRS $1915+105$ is in agreement with the prediction of the 3:2 parametric epicyclic resonance model, but the same prediction for GRO 1655-40 does not match the spectral fitting. No particular resonance model considered so far can cover the spectral limits to the spin for both microquasars. It could be interesting that the recently proposed 3:2 periastron precession resonance (Bursa 2005b) implies the spin of GRO 1655-40 to be $a \sim 0.7$. Nevertheless, eventuall periastron precession resonance requires the spin of GRS $1915+105 a<0.8$ which is in strong disagreement with the spectral fitting limit, $a>0.98$.

\section{Troubles with the spin: $1 / M$ scaling}

In principle one cannot exlude the possibility of different mechanisms exciting the high frequency QPOs in different sources, but there are many indicies that the mechanism is the same or similar (e.g., van der Klis 2005; Török et al. 2006). McClintock \& Remillard (2003) found that the upper QPO frequency in microquasars scales well as $\nu_{U}=2.793\left(M_{0} / M_{\odot}\right)^{-1} \mathrm{kHz}$ which is in good agreement with the $1 / M$ scaling of the first term in equation (1.1).

On the other hand the exact $1 / M$ scaling holds only for the fixed value of the spin $a$ as functions $f_{\mathrm{i}}$ in equation (1.1) are sensitive to the spin. The spectral limits to the spin for the two microquasars are very different: $a \sim 0.7$ vs. $a>0.98$, and, in addition,

$\dagger$ Because of the properties of Kerr black hole spacetimes, any relativistic model of black hole QPOs should be rather sensitive to the spin $a$, however this sensitivity can be negligible on large scales of mass (Abramowicz et al. 2004). 
functions $f_{\mathrm{i}}(a, x)$ are more sensitive to the value of the spin when it is close to $a=1$ (e.g., Török \& Stuchlík 2005). Hence, if the spin values obtained from the spectral fits are correct, the observed high frequency QPOs do not show sensitivity to the spin $a$ under the assumption of a unified QPO model. This is a serious problem for any relativistic QPO model handling with the orbital and epicyclic frequencies (1.1).

\section{Requirement of a more realistic description}

It was found recently that the pressure effects may have a strong influence on the oscillation frequencies. Šrámková (2005) and Blaes et al. (2006) studied properties of the radial and vertical epicyclic modes of slightly non-slender tori within Newtonian theory using the Paczyński-Wiita potential, and found the epicyclic frequencies to decrease with increasing thickness of the torus. The same behaviour was found for the resonant radius where the frequencies are in a 3:2 ratio, which on the contrary implies increase of the resonant frequencies. Considering the appropriate corrections to frequencies in the Kerr metric, one can reestimate the values of the spin using the resonance model. If the results in the Kerr metric were following the same trend as those in the Paczyński-Wiita case, the spin for some configurations can be lower than previously estimated. $\dagger$

\section{Acknowledgements}

This research is supported by Czech grant MSM 4781305903.

\section{References}

Abramowicz, M.A. \& Kluźniak, W., 2001, A\&A 374L, 19A, astro-ph/010507

Abramowicz, M.A. \& Kluźniak, W., 2004, AIP Conference Proceedings, Vol. 714, Edited by Kaaret, Philip, Frederick, K. Lamb, and Swank, Jean H., Melville, NY: AIP, 2004, p.21-28

Abramowicz, M.A., Kluźniak, W., McClintock, J.E. \& Remillard, R. A., 2004, ApJ 609, L63

Aliev, A.N. \& Galtsov, D.V., 1981, Gen. Relativity Gravitation, 13, 899

Beer, M.E. \& Podsiadlowski, P. 2002, MNRAS 331, 351

Blaes, O.M., Šrámková, E., Abramowicz, M.A., Kluźniak, W. \& Torkelsson, U., 2006, ApJ, submitted

Bursa, M., in Proceedings of RAGtime 6/7: Workshops on black holes and neutron stars, Opava, 16-18/18-20 September 2004/2005, edited by S. Hledík and Z. Stuchlík, Silesian University in Opava, Opava, 2005b, ISBN 80-7248-242-4

Greene, J., Bailyn, Ch. D. \& Orosz, J.A., 2001, ApJ 554, 1290

Horák, J., 2005, Astronomische Nachrichten 326, 845-848

Kluźniak, W., \& Abramowicz, M.A., 2000, Phys. Rev. Lett., submitted, astro-ph/0105057

Kluźniak, W., Abramowicz, M.A., 2002, astro-ph/0203314

McClintock, J.E. \& Remillard, R.A., 2003, astro-ph/0306213

McClintock, J.E., Shafee, R., Narayan, R., Remillard, R.A., Davis, S.W., Li, L.X., 2006, accepted for publication in ApJ, astro-ph/0606076

Nowak, M.A. \& Lehr, D.E., 1998, in Theory of Black Hole Accretion Disks, ed. M.A. Abramowicz, G. Björnsson, \& J.E. Pringle (Cambridge: Cambridge University Press), 233-253

Šrámková, E., 2005, Astronomische Nachrichten, 326, 835-837

Török, G., 2005, Astronomische Nachrichten, 326, 856

Török, G., Abramowicz, M.A., Kluźniak, W. \& Stuchlík, Z., 2005, A\&\&A, 436, 1

Török, G. \& Stuchlík, Z., 2005, A\&A, 37, 775-788

Török, G., Abramowicz, M. A., Kluźniak, W. \& Stuchlík, Z., 2006, in print, astro-ph/0603847 van der Klis, M., 2005, Astronomische Nachrichten, 326, 798-803

$\dagger$ In case of the 3:2 parametric resonance, the maximal realistic increase of the resonant frequency due to the pressure effects is about 15 percent (Blaes et al. 2006), which for GRO $1655-40$ and the mass estimate by Beer \& Podsiadlowski lowers the spin down to $a \sim 0.8$. 